\title{
Os EUA, a exportação e a expansão do ensino de Management no Brasil nas décadas de 1950 e 1960
}

RAFAEL ALCADIPANI

Fundação Getulio Vargas / Escola de Administração de Empresas de São Paulo, Departamento de Administração Geral E RECURSOS HUMANOS, SÃo PAULO - SP, BRASIL

Carlos Osmar Bertero

Fundação Getulio Vargas / Escola de Administração de Empresas de SÃo Paulo, Departamento de Administração Geral e RECURSOS HUMANOS, SÃo PAULO - SP, BRASIL

\begin{abstract}
Resumo
Estudos a respeito da história do ensino de Administração têm ganhado destaque no campo, recentemente, em nosso país. Neste artigo, discutimos como instituições dos EUA atuaram na exportação e expansão do ensino de Management no Brasil durante os anos de 1950 e 1960. Argumentamos que a representação diplomática dos EUA, a Universidade do Michigan e a Fundação Ford formaram uma rede que possibilitou a exportação e expansão do ensino do Management no País. Este artigo contribui por aprofundar a discussão sobre a exportação e expansão do ensino do Management para o Brasil, e por contextualizar essa ação dentro das relações Brasil-EUA da época. Além disso, mostramos detalhadamente o papel das instituições norte-americanas nesse processo e colocamos ainda a expansão do ensino do Management no Sul e no Nordeste do Brasil como resultado de apoio e pressões por parte dos EUA.
\end{abstract}

Palavras-chave: Management. Educação. Relações Internacionais.

\section{The USA and the export and expansion of Management education in Brazil in the 1950s and 1960s}

\section{Abstract}

Studies on the history of management education have gained preeminence in the field of organizational studies recently in Brazil. In this article we discuss how U.S. institutions exported and expanded management education in Brazil during the 1950s and 1960s. We argue that the U.S. diplomatic representation, Michigan State University and Ford Foundation have formed a network that enabled the exportation and expansion of management education in Brazil. This article contributes to deepen discussions on the issue of exports and expansion of management education in Brazil and to offer a context of this issue within Brazil-US relations. In addition, the study shows details on the role of North American institutions in this process and the expansion of management education in the South and the Northeast of Brazil as the result of support and pressures from the USA.

Keywords: Management. Education. International Affairs.

\section{EUA, la exportación y la expansión de la enseñanza del Management en Brasil en las décadas de 1950 y 1960}

\section{Resumen}

Los estudios de la historia de la enseñanza de administración, han recientemente adquirido relevancia en el área, en nuestro país. En este artículo, discutimos cómo las instituciones de los Estados Unidos actuaron en la exportación y expansión de la enseñanza del Management en Brasil durante los años 1950 y 1960. Argumentamos que la representación diplomática de EUA, la Universidad de Michigan y la Fundación Ford formaron una red que posibilitó la exportación y expansión de la enseñanza del Management en el País. Este artículo contribuye para profundizar la discusión sobre la exportación y expansión de la enseñanza del Management en Brasil, y también para contextualizar esa acción dentro de las relaciones Brasil-EUA de la época. Además, mostramos detalladamente el papel de las instituciones estadounidenses en ese proceso y también colocamos la expansión de la enseñanza del Management en el sur y en el nordeste de Brasil como resultado del apoyo y de las presiones de EUA.

Palabras clave: Management. Educación. Relaciones Internacionales. 


\section{INTRODUÇÃO}

Este é um estudo a respeito da sociologia da ciência da Administração, uma vez que aborda a história do campo, e está circunscrito a análises da importação do Management para o Brasil (SERVA, 1992). No final do século XIX e início do século XX, o management despontou como um conjunto de técnicas e habilidades que poderiam ser ensinadas no sistema escolar. É, também nesse período, que a profissão de administrador emerge. Em inúmeros países da Europa, o ensino de Administração não surgiu dentro de universidades, mas, sim, com a criação de escolas comerciais independentes no final dos anos de 1800 e início de 1900 (ÜSDIKEN e ÇETIN, 2001). Porém, é nos Estados Unidos da América (EUA) que o ensino de management transforma-se em um curso universitário e ganha destaque mundial. Conforme destacam Alcadipani e Rosa (2011), modos de organizar fazem parte de nossa civilização desde suas origens, todavia, é em um contexto histórico e econômico específico que uma maneira de administrar particular emerge. Tal maneira surge nos EUA e preza pela eficácia, eficiência, maximização de resultados e, sobretudo, pretende-se politicamente neutra. É exatamente isso que o Management significa (ALCADIPANI e ROSA, 2011).

A consolidação do ensino do Management ocorreu nos EUA no contexto da emergência e consolidação das grandes corporações e com a intenção de se construir um corpo profissional para assumir o seu comando (KHURANA, 2007). Para Khurana (2007), a figura do gestor não se encontrava na visão econômica convencional, na qual apenas se reconheciam o proprietário e o trabalhador. As funções do gestor, atualmente reconhecidas e socialmente aceitas, eram relacionadas tão somente a direção de pessoas, definição de procedimentos e formas de vender produtos, estabelecimento das maneiras de distribuir o que se produzia, entre outras atividades. Ao longo da realização de tais funções, os gestores começaram a se apropriar gradualmente de tarefas até então exercidas por empreendedores. É nesse contexto que surgem as escolas de negócios nos Estados Unidos, que tiveram papel preponderante não apenas em formar profissionais para assumir o comando das corporações, mas, também, funcionaram como instrumento fundamental na criação da profissão de gestor (KHURANA, 2007).

A consolidação do ensino de Management coincidiu com o estabelecimento dos Estados Unidos como superpotência mundial no século XX (BERTERO, 2006), e o que é conhecido atualmente como gestão é, fundamentalmente, considerado como uma criação norte-americana. $O$ ensino de Management dos EUA difundiu-se no mundo por meio da americanização, um processo deliberado e consciente de difusão dos valores básicos dos EUA em países sob sua influência, como resultado de sua preponderância política, econômica e militar (ALCADIPANI e CALDAS, 2012; GERSTLE, 1989; TOTA, 2000). Na América Latina, a criação de instituições com ensino de Management ancorou-se em um modelo norte-americano (CALDAS, 1997 CALDAS e ALCADIPANI, 2006; GANTMAN e RODRIGUES, 2008). Na Argentina, por exemplo, o primeiro curso de graduação em Management foi criado em 1958 na Universidade de Buenos Aires. Para ministrar as diversas disciplinas, professores daquela escola foram encaminhados para estudar na Universidade de Columbia, nos Estados Unidos.

Ao longo de seus quase 60 anos de existência, o ensino de Management no Brasil assumiu dimensões impressionantes. Em mais de 2000 cursos de graduação no País, quase $20 \%$ dos alunos estão matriculados em um curso de Management. Há, ainda, mais de uma centena de cursos de pós-graduação stricto sensu reconhecidos pela Coordenadoria de Aperfeiçoamento de Pessoal de Nível Superior (CAPES); e, aproximadamente, 200.000 professores na área atuam apenas nos cursos de graduação. Existe, ainda, um número significativo de cursos de pós-graduação lato sensu a respeito dos quais não se conhecem estatísticas confiáveis, mas que se estima ser próximo de uma dezena de milhar. Poucos estudos foram realizados com o intuito de entender as origens históricas de tal expansão.

O ensino do Management no Brasil teve início na cidade de São Paulo, que já despontava como foco de crescimento econômico e base industrial no final dos anos 1940. O primeiro curso foi ministrado na Escola Superior de Negócios (ESAN) por iniciativa do padre jesuíta Roberto Sabóia de Medeiros, que mantinha relações com a Universidade de Harvard. Apesar do pioneirismo, a ESAN perdeu a proeminência na difusão do ensino de Management no Brasil para a Escola de Management de São Paulo da Fundação Getulio Vargas (FGV EAESP) e para a Faculdade de Economia e Management da Universidade de São Paulo (FEA-USP). Estudos recentes analisaram a criação da EAESP-FGV (ALCADIPANI e BERTERO, 2012), da FACE-UFMG (BARROS, 2013) e do departamento de Management da FEA-USP (VALE, 2012).

No caso da criação e desenvolvimento da FGV EAESP, os EUA, principal financiador da difusão do ensino de Management no mundo, tiveram papel de destaque na medida em que ajudaram a financiar essa escola, participando com o envio de uma missão universitária de professores da Michigan State University (MSU) para criar a instituição, além de fornecer treinamento e formação em instituições de ensino dos EUA a professores brasileiros. Professores da MSU estiveram presentes ainda na UFRGS e na UFBA, denotando a presença importante de instituições norte-americanas na exportação e expansão do ensino no Management no Brasil. Neste artigo, analisamos o papel desempenhado pelos EUA na exportação e expansão do ensino de Management 
no Brasil, durante as décadas de 1950 e 1960, em especial na criação dos cursos de Management na UFBA e na UFRGS. Assim, nossa questão orientadora neste texto é: como instituições dos EUA atuaram na expansão do ensino de Management no Brasil durante os anos de 1950 e 1960, em especial nos casos dos cursos de Management das Universidades Federais da Bahia e do Rio Grande do Sul? Escolhemos os cursos da UFRGS e da UFBA por terem sido os pioneiros no Sul e no Nordeste do Brasil, respectivamente, e, por isso, serem cruciais à expansão do ensino da gestão nessas importantes regiões do Brasil.

\section{METODOLOGIA: HISTORIOGRAFIA E PESQUISA EM ARQUIVO}

A História é um campo de conhecimento amplo e diverso, dotado de inúmeras correntes e abordagens teóricas (IGGERS, 1997; WARREN, 1998) que o tornam repleto de complexidades. Neste artigo, tomamos História como uma abordagem de pesquisa, ou seja, ela será aqui considerada como um método. Dessa forma, pretendemos realizar uma historiografia (MILLS e YUE, 2011), algo que já foi realizado por outros autores da área de estudos organizacionais (por exemplo, ALCADIPANI e BERTERO, 2011; ALCADIPANI e BERTERO, 2012; BOOTH e ROWLINSON 2006; COOKE, 1999; VIZEU, 2010). Todavia, no Brasil, apesar de crescentes, ainda são incipientes os estudos ligados à história do Management e das organizações. Exemplos desses estudos são o de Segnini (1982), que discutiu mecanismos de poder em operação na Companhia Paulista de Estradas de Ferro; de Vizeu (2008), que analisou a formação do Instituto de Organização Racional do Trabalho (IDORT); e de Curado (2005), que analisou documentos e realizou entrevistas a fim de discutir a emergência e formação dos saberes em Management no País.

No que diz respeito ao ensino do Management, destacam-se alguns estudos que mencionamos a seguir. Fischer (1984), por meio de entrevistas e análise de documentos, discutiu o desenvolvimento do ensino brasileiro de Administração Pública. Covre (1981), na análise de documentos, entrevistas e questionários, percebeu a EAESP como uma instituição cujo objetivo fundamental é atender ao capital. Financiado pela Fundação Ford e por meio de análise de documentos, Anderson (1987) discutiu o desenvolvimento da educação em Management no Brasil. Vale frisar que o citado autor fez parte da missão da MSU, a qual criou a EAESP. Alcadipani e Bertero (2012) mostraram o papel desempenhado pela Guerra Fria na criação da EAESP-FGV e, em outro texto, os autores mostram como a EAESP FGV é uma instituição híbrida, não uma mera cópia de escolas de Management dos EUA (ALCADIPANI e BERTERO, 2012). Vale (2012) fez um estudo sobre a criação do departamento de Management da FEA-USP. Barros (2013) analisou a história da FACE-UFMG e Barros e Carrieri (2013) mostraram o papel dos convênios de cooperação Brasil-EUA nos anos de 1940 e 1950 na difusão do ensino de Management no Brasil. Existem, ainda, livros em tom triunfalista que apresentam depoimentos a respeito da criação de algumas instituições de ensino e pesquisa na área (D’ARAUJO, 1999).

É possível argumentar que há um crescente interesse pela historiografia no campo de Teoria das Organizações e do Management (BOOTH e ROWLINSON, 2006; CLARK e ROWLINSON, 2004; COSTA, BARROS e CARVALHO, 2011; COSTA, BARROS e MARTINS, 2010; DUREPOS, MILLS e WEATGERBEE, 2012; VIZEU, 2010;). Dentre as diferentes correntes, este artigo afilia-se ao campo emergente de História do Management e das Organizações (BOOTH e ROWLINSON 2006), o qual se diferencia da História dos Negócios uma vez que nele as ideias, práticas e discursos do Management são consideradas como fenômenos históricos, sociais e geopolíticos. Nesse campo emergente, defende-se, ainda, que estudos históricos dizem respeito ao presente e ao passado concomitantemente (COOKE, 2003), e, além disso, considera-se que a maneira pela qual a história é escrita permite selecionar e ignorar eventos passados, sendo tais escolhas moldadas pelas presentes relações de poder e suas ideologias associadas (COOKE, 1999).

Para Cooke (2003), aquilo que é considerado como historiografia contribui para a legitimação das instituições, práticas e corpos de conhecimento contemporâneos. Essa abordagem permite-nos mostrar que o presente não é algo natural, mas, sim, o resultado de ações e práticas específicas que poderiam ser outras (JACQUES, 2006). Além disso, nesta pesquisa, partiremos do pressuposto de que o objeto de análise somente pode ser compreendido dentro dos contextos histórico nacional e internacional de sua época. Isso implica que as organizações e o conhecimento gerado sobre elas são tratados como algo imerso em um contexto histórico-social, o qual precisa ser investigado e analisado (MARCH, 2007).

A historiografia está tradicionalmente relacionada à análise de documentos (COSTA, BARROS e MARTINS, 2010), os quais se constituem em arquivos. Para Mills e Mills (2011, p. 342), arquivos são:

The materials in archive collections were collected for a variety of reasons, ranging from the filing of documents for marketing, legal, or other business-related requirements through to the establishment of a historical record. In the former cases the collected materials do not formally constitute an archive but the term is often applied by researchers who access them for the purpose of studying a particular 
phenomenon, institution, group, place or person. It is usually once these kinds of materials are specifically collected together for the purpose of preserving a 'history' of an organization, person, or place, and are 'housed' in a distinct location that they become formally designated an 'archive'.

Neste estudo, analisamos os relatórios enviados pela missão de professores da Michigan State University - incumbida de criar a FGV EAESP - em razão de estes terem desempenhado papel crucial na difusão do ensino de Management para outras escolas do Brasil. Tivemos acesso a esses relatórios, que estavam localizados no arquivo da presidência da FGV, no Rio de Janeiro. Analisamos, ainda, documentação da Fundação Ford a respeito do mesmo período, uma vez que essa fundação também apoiou a difusão do ensino de Management no Brasil, no período de análise. Esses documentos foram coletados na sede da Fundação Ford, em Nova lorque, nos Estados Unidos. Por fim, analisamos telegramas - disponíveis na internet - enviados pelos embaixadores dos EUA no Brasil para o Departamento de Estado. Por fim, coletamos e analisamos arquivos presentes nos arquivos históricos da MSU.

É preciso lembrar que a especificidade dos fenômenos em nosso contexto nacional é bastante diversa daquela produzida nos países onde o Management surge como uma disciplina organizada, uma corrente de pensamento e uma prática social, o que revela a emergência de uma historiografia do Management brasileiro. A seguir, buscamos o papel das instituições norte-americanas na difusão do ensino de Management no Brasil. Focamos nas décadas de 1950 e 1960 pelo fato de ser o momento em que o ensino de Management deixa de ser uma exclusividade da cidade de São Paulo e passa a ser ensinado também no Rio Grande do Sul e para a Bahia.

\section{INSTITUIÇÕES DOS EUA E A EXPANSÃO DO ENSINO DE MANAGEMENT NO BRASIL}

\section{Representação diplomática dos EUA}

O envio do ensino do Management para o Brasil estava ligado a programas de ajuda dos EUA para outros países do mundo no cenário de Guerra Fria. Especificamente a vinda do Management para o Brasil está circunscrita a uma lógica de diversos acordos de cooperação entre o Brasil e os EUA que aconteceram nos anos de 1950 e 1960. Esses esforços de ajuda faziam parte das atividades do Ponto IV e, posteriormente, da USAID, ambos órgãos do governo norte-americano. A relação entre a Guerra Fria, o Ponto IV e o ensino do Management no Brasil já é conhecida e foi analisada em outro texto (ALCADIPANI e BERTERO, 2011).

Por se tratar de um projeto que envolvia o governo dos EUA, a representação diplomática do país no Brasil desempenhou um papel importante em negociações que estavam indiretamente relacionadas com a exportação e a expansão do Management dos EUA para o Brasil. Essa exportação e expansão estão imersas no contexto das relações entre Brasil e EUA, que possuem representação diplomática no País desde outubro de 1825. Nos anos de 1950 e 1960, os EUA tiveram nove diferentes embaixadores no País. O Quadro 1 mostra a lista de embaixadores e o tempo em que serviram no Brasil.

\section{Quadro 1}

\section{Embaixadores norte-americanos no Brasil}

\begin{tabular}{|c|c|c|}
\hline Nome do embaixador & Data de início & Data de término \\
\hline Herschel Vespasian Johnson & 22 de julho de 1948 & 27 de maio de 1953 \\
\hline James S. Kemper & 18 de agosto de 1953 & 26 de julho de 1955 \\
\hline James Clement Dunn & 11 de março de 1955 & 04 de julho de 1956 \\
\hline Ellis O. Briggs & 24 de julho de 1956 & 29 de abril de 1959 \\
\hline Clare Boothe Luce & 29 de abril de 1959 & 01 de maio de 1959 \\
\hline John M. Cabot & 22 de julho de 1959 & 17 de agosto de 1961 \\
\hline Lincoln Gordon & 19 de outubro de 1961 & 25 de fevereiro de 1966 \\
\hline John W. Tuthill & 30 de junho de 1966 & 09 de janeiro de 1969 \\
\hline Charles Burke Elbrick & 14 de julho de 1969 & 07 de maio de 1970 \\
\hline
\end{tabular}

Fonte: Elaborado pelos autores. 
O contrato que celebra o acordo entre o governo dos EUA e a MSU é de junho de 1953. A análise dos telegramas dos embaixadores dos EUA para o governo do seu próprio país revela que, entre 1952 e 1956, a missão diplomática dos EUA esteve envolvida em expressivas controvérsias com o governo brasileiro, as quais estavam diretamente relacionadas com a cooperação técnica e financeira entre EUA e Brasil. Após 1956, tais controvérsias e disputas diminuíram de forma significativa, possivelmente devido à estabilização da situação do Brasil com o governo de Juscelino. Quando da renúncia de Jânio Quadros, a questão política vira tema central das conversas, e a cooperação técnica e econômica não fica tanto em evidência.

Há diferentes telegramas que sugerem a relação conturbada entre Brasil e EUA daquela época. Por exemplo, no dia 11 de março de 1952, às 8h00, o embaixador dos EUA, Johnson, enviou telegrama classificado como secreto para o Departamento de Estado, relatando uma conversa que teve com o Ministro das Relações Exteriores do Brasil, nomeado no texto como FonMin.

There are two groups in Braz whose activities and intentions are hostile to US-Braz collaboration. One of these groups is Commie whose motives are known and require no particular discussion relation present situation. Other group composed people who profess profound skepticism at practical possibility of any benefit to result to Braz through coop with US. This group at present moment is focusing its attacks on Lafer who is staunch proponent Braz-US collaboration. Any indication that banks are withholding action on Joint Comm projects as means of pressure on Braz Govt would be according to FonMin immed pointed to by Lafer's enemies as proof that US lacks sincerity and that loans so confidently promised by Lafer wld never materialize. [...] FonMin further stated that if Lafer shld lose out in his present fight, he wld unquestionably be replaced by some one not favorable to close coop with US, that it wld mean a serious setback for all proponents of that collaboration. He considers it particularly unfortunate that this crisis has arisen just at moment when US and Braz are approaching agreement on various important matters long under study. [...]. While he did not specifically say so, he indicated that a polit crisis which wld result in Lafer's departure might jeopardize or seriously retard our agreement on other matters.. (832.00 TA/3-1152: TELEGRAM).

O telegrama deixa claro que o então Ministro da Fazenda, Horácio Lafer, era a favor da colaboração com os EUA, porém, enfrentava resistências de outros grupos, entre os quais, comunistas (commie, conforme mencionado acima). Indicava, ainda, que, caso os bancos dos EUA decidissem interromper o fluxo de recursos como forma de pressionar o governo brasileiro em torno de uma disputa que o Brasil e os bancos dos EUA estavam travando, a posição de Lafer no governo de Getulio Vargas seria ainda mais enfraquecida. A saída de Lafer é percebida como prejudicial às relações entre Brasil e EUA, pois, possivelmente, poderia afetar as negociações ligadas ao auxílio dos EUA para a exportação e expansão da educação em Management no Brasil.

Em outro telegrama enviado para o Departamento de Estado, às 8 h00 do dia 09 de maio de 1952, o Embaixador Johnson relata um encontro em que esteve com o Presidente Getúlio Vargas.

Began his own remarks with statement that he hoped we wld realize tremendous pressure now on him through growing force of nationalism, which he frankly said had been linked in many minds with Commie, but nevertheless, was a major factor in his own political calculations. He spoke of resentful feeling existing in Braz because of large sums of money allocated through Marshall Plan to Eur, and other areas of the world in comparison to what had been done for Braz. He also expressed his disappointment and dissatisfaction with slow progress made by Joint Comm (800.05132/5-952: TELEGRAM).

No telegrama, o embaixador destaca a posição expressa pelo presidente Getulio Vargas de que os comunistas estavam buscando influenciar seu governo. Fica ainda expresso o ressentimento do governo brasileiro pela suposta falta de atenção por parte dos EUA ao país, especialmente quando comparada à recebida por Europa e outras partes do mundo. Menciona, também, a insatisfação de Vargas com os progressos feitos pela EUA-Brazil Joint Economic Development Commission, órgão que iria organizar e gerir os programas de cooperação técnica e econômica entre os países. Vale lembrar que, antes da II Guerra Mundial, o Governo dos EUA já havia estabelecido parcerias de auxílio para o Brasil. Habilmente, Getulio Vargas conseguiu atrair acordos de cooperação técnica e financeira desde o início do seu primeiro governo (TOTA, 2000). O Presidente Café Filho, que ocupou o cargo por pouco mais de um ano após o suicídio de Getulio Vargas, insistia na necessidade de cooperação entre os dois países. Em telegrama enviado para o Departamento de Estado, em 7 de Janeiro de 1955, às 7h00, o Embaixador Kemper relatou e comentou o encontro com o Presidente do Brasil à época:

Pledge of assistance to friendly nations plus continued press references to Marshall Plan for aid has caused Brazilians to ask In what respects have we failed to cooperate and why is assistance we so 
desperately need being denied us?" Actually Brazil has not acted with reasonable promptness on a number of pending matters. But the heroic effort being made to bring some order out of present chaotic financial situation merits both our admiration and our assistance" (732.00/1-755).

Mais uma vez a questão do Plano Marshall vem à tona e o governo brasileiro parece ressentido com o que consideraria falta de apoio dos EUA. O Embaixador recomenda que os EUA auxiliem o Brasil naquele momento, pois o governo norte-americano mostrava que estava tentando resolver a situação do Brasil. Em telegrama confidencial enviado em 24 de janeiro de 1955, o Embaixador Kemper comenta sobre o governo de Café Filho:

From the United States standpoint, the most serious development is the growing inclination to place the blame on the United States for not having moved rapidly and generously with respect to financial aid and to ascribe to this lack of outside aid the responsibility for the Administration's failure to supply courageous leadership. This viewpoint is beginning to take root among Brazilians who are normally our staunch supporters. Officials of the Government are discouraged and are tending toward a frame of mind which may prejudice the attainment of United States objectives and aid the election of individuals who may pose future serious problems for United States policy. [...] In spite of its weaknesses, the Café Filho Government is fundamentally well disposed toward the United States, which scarcely could be said of its predecessor, and has tried to place its nation's welfare above personal interests or petty political ambitions (732.00/1-2455).

Fica evidente uma atmosfera de animosidade contra os EUA no Brasil, que vinham sendo acusados até mesmo de não prestar assistência ao País quando parecia necessário. Há, ainda, a indicação de que Vargas era pouco amistoso com relação aos EUA. Com a eleição do governo de Juscelino Kubitschek, o embaixador dos EUA da época visita o presidente eleito em outubro de 1955 para saber se comunistas teriam importância em seu governo. O Embaixador Dunn relatou ao Departamento de Estado que:

He stated that he was not a Communist would not permit the Communists to be active in his government and recalled that he when Deputy for Minas, had voted to deprive the Communist Party of legal status. He regretted that certain of the press in US were giving impression he was connected with the Communists and he was concerned that such an impression might interfere with the good relations he hoped to have between Brazil and the US (732.00/10-1955).

Por meio dessa comunicação fica explícita também uma grande disposição dos EUA de colaborar com o então eleito presidente do Brasil, desde que ele não estivesse sob influência comunista. De uma forma geral, os telegramas enviados pelo embaixador dos EUA para o Departamento de Estado, de 1953 a 1956, época crucial à exportação e expansão do ensino do Management para o Brasil, deixam claras as dificuldades enfrentadas pelo País em seu cenário econômico e social. Indica ainda que há um contexto de relações conturbadas entre Brasil e EUA.

Periodicamente a Embaixada dos EUA realizava, por sua vez, leituras sobre a situação brasileira, as quais davam subsídios ao governo daquele país em decisões relativas aos seus interesses no Brasil. Olhavam com muita desconfiança quando um novo político assumia a Presidência, em especial, a forma com que iria se relacionar com os EUA levando em conta os interesses norte-americanos no País, e a relação que teria com os comunistas. Além disso, constata-se que a Embaixada produzia guias das cidades brasileiras para serem enviados a professores dos EUA que vinham ao Brasil. Há guias e comentários de cidades como Porto Alegre, São Paulo, Salvador e Rio de Janeiro. Por fim, os consulados em São Paulo, Porto Alegre e Salvador serviam para os serviços de correio entre os membros da MSU no Brasil e a sua base nos EUA. Os relatórios do progresso da missão eram encaminhados por meio da correspondência diplomática. Além disso, a representação diplomática dos EUA cumpria as funções consulares para os professores norte-americanos baseados no Brasil. A representação diplomática dos EUA exerceu, assim, uma função de apoio e esteve mais distante da importação e expansão do Management para o Brasil. A MSU e o Institute of Inter-American Affairs tiveram um papel mais proeminente, conforme analisaremos a seguir.

\section{O INSTITUTE OF INTER-AMERICAN AFFAIRS E A MICHIGAN STATE COLLEGE OF AGRICULTURE AND APPLIED SCIENCES}

Em 29 de junho de 1953, é firmado o contrato IAAc-45 entre o Governo dos EUA, representado pelo The Institute of InterAmerican Affairs - the Technical Cooperation Administration (IIA) e a MSU, então denominada como Michigan State College of 
Agriculture and Applied Sciences. O contrato versa sobre o apoio que a MSU daria à FGV no estabelecimento e na condução de uma escola de negócios no Brasil. A função do IIA era promover e aumentar a solidariedade hemisférica Sul e a cooperação interamericana, especialmente nas áreas comercial e econômica. O IIA surgiu na década de 1940 como uma maneira de auxiliar na construção de uma imagem favorável dos EUA na América Latina, em um contexto de pré-II Guerra Mundial, e com o intuito de afastar a influência do Eixo sentida no subcontinente. O IIA cumpriu papel importante na americanização do Brasil, constituindo-se como uma "fábrica de ideologias" (TOTA, 2000). Na década de 1950, o instituto estava menos influente no Brasil, mas, mesmo assim, foi a agência utilizada pelo governo dos EUA para conduzir a exportação do Management ao País.

O contrato IAAc-45 estabeleceu que a MSU deveria alocar, no Brasil, "técnicos" qualificados no campo de gestão seguindo a direção e a coordenação do diretor da missão do IIA no País. A função fundamental era a de assessorar, aconselhar e assistir a FGV na criação, estabelecimento e desenvolvimento de uma escola de negócios. O contrato estabelecia que o IIA pagaria por ano US\$32.904 para a MSU por suas atividades, valor equivalente, atualmente, a cerca de US\$ 700.000 (http://www.measuringworth.com/uscompare/relativevalue.php). O montante deveria ser utilizado para o pagamento de salários e demais despesas dos "técnicos" da MSU enviados ao Brasil, e 12,5\% alocados para cobrir os custos administrativos da MSU com a sua operação nos trópicos. A MSU tinha de enviar os comprovantes de despesa para o IAA como forma de prestar contas de seus gastos.

O IIA comprometeu-se a conseguir, para a missão da MSU no Brasil, por meios próprios ou com o apoio do Governo do Brasil, o seguinte: (a) espaço em escritórios adequados, equipamentos de escritório e assistência de secretários e secretárias e demais pessoal administrativo; (b) transporte local para os membros da missão dentro do Brasil quando desempenhando atividades ligadas ao trabalho na missão; e (c) equipamentos, materiais e todos os demais recursos necessários para o desempenho da missão no Brasil. Dessa forma, a IIA estabeleceu-se como a gestora e a organização que financiaria a exportação e a expansão do Management ao País.

Após 18 anos de Michigan ter se transformado em um estado, foi criado no dia 15 de fevereiro de 1855 o Agricultural College of the State of Michigan, instituição que mais tarde se transformaria na MSU. Em seu início, o College ministrava cursos na área de Ciências Agrárias - foi a primeira instituição no país a ensinar ciências da agricultura. Com o passar do tempo, novos cursos foram estabelecidos.

A MSU teve um papel fundamental no ideal norte-americano de levar o "progresso" ao mundo. Horn (1985) fez um levantamento dos projetos de desenvolvimento internacional entre 1951 e 1985, período em que houve a participação da MSU. A autora contabilizou 150 projetos em que a MSU esteve envolvida no exterior. Exemplos desses projetos são: polícia e gestão pública no Vietnã; expansão da universidade do Zimbábue; revisão do ensino de agricultura na Tailândia; auxílio no desenvolvimento da indústria cinematográfica no Irã; desenvolvimento de capacitação em agricultura na Indonésia; estabelecimento da Universidade de Addis Abba, na Etiópia; somente para citar alguns. A MSU forneceu apoio e capacitação técnica em inúmeras áreas de conhecimento em praticamente todos os continentes do mundo.

Em um contexto de Guerra Fria, o aumento da presença de norte-americanos no exterior em diferentes missões técnicas e de cooperação bilateral como forma de se opor à URSS (ALCADIPANI e BERTERO, 2011) levou ao desenvolvimento de uma série de ações no sentido de facilitar essa presença. Foram produzidos, por exemplo, diversos materiais a respeito do comportamento adequado daqueles que, estando fora do país, o representariam no exterior. Uma Organização Não Governamental, American Council for Nationalities Service emitia materiais informativos para serem entregues aos profissionais dos EUA que faziam parte de missões no exterior. Os "técnicos" da MSU que vieram ao Brasil receberam esse tipo de material e treinamento. Em uma brochura de 1959, "Americans Abroad Spokesmen for the United States", consta uma introdução feita pelo então Presidente Eisenhower:

Year after year increasing numbers o four citizens travel to foreign countries. In mosto $f$ these lands there exists a reservoir of good will for the United States and a knowledge of what we stand for. In some áreas our country aspirations are less well understood. To all varied peoples of these many countries, the American citzen abroad represents the United States of America.

O documento começa informando: "representing our country overseas is a pretty big assignment". A brochura mostra os "Dos e os Don'ts of international conversation" e apresenta respostas para perguntas difíceis. Exemplos de questões: "Do the Americans really want peace? Isn't U.S. talk about a position of strenght evidence of your aggressive intentions? "Why does the U.S. maintain a ring of bases around the Soviet bloc? Wasn't the U.S. guilty of aggression in sending its Lebanon? Does the U.S. really want disarmament? Isn't true that U.S. policies tend to devide the world into hostile camps?" 
A minúcia das indicações sobre o modo como se portar chegava até mesmo às esposas. Havia documentos a respeito de como as esposas dos americanos deveriam se comportar no exterior. Um deles, de 1962, começa assim:

About a quarter of a million American wives are now living abroad with their husbands who work for our government, business firms, churches, and foundations. The way they live has suddenly become a national concern. What happens to an air base, an aid program or a missionary school may largely depend on them.

No Brasil, há registros de quatro projetos desenvolvidos pela MSU. O primeiro deles, financiado pela USAID, durou de 1960 a 1965 e teve como objetivo criar um centro audiovisual para o desenvolvimento de material de ensino e treinamento para escolas e universidades. O centro foi desenvolvido dentro da Universidade de São Paulo. O segundo projeto, também financiado pela USAID, tinha o objetivo de expandir e melhorar o ensino em ciências agrárias no País - durou de 1974 a 1978. Houve a participação da USP, UFRGS, UFV, UFMG. UFCE e a UFRRJ. O terceiro projeto aconteceu entre os anos de 1958 e 1965, seu objetivo era institucionalizar o ensino de gestão no Brasil - foi financiado pela Ford Foundation e pelo governo brasileiro. Horn (1985) informa que participaram desse projeto a UFBA, a UFRGS e as universidades de Indiana, Syracuse e Pittsburg, muito embora não tenhamos encontrado outras menções a essas universidades em outros documentos. Por fim, houve a criação das escolas de Management, que duraram de 1953 a 1966. O projeto envolveu a EAESP, a UFBA e a UFRGS, com um custo de $\$ 799.107$ (valor que hoje equivaleria a cerca de U\$ 10 milhões, de acordo com http://www.measuringworth.com/uscompare/relativevalue.php) e foi financiado pelo governo brasileiro e pela USAID.

Dessa forma, a exportação e a expansão do Management para o Brasil fizeram parte de um grupo extensivo de projetos de desenvolvimento financiados e apoiados pelos EUA ao redor do mundo. Além disso, no caso brasileiro, a MSU não estava apenas envolvida com Management, mas, também, com a agricultura e capacitação técnica em audiovisual para a produção de materiais didáticos. Ao levar em conta o contexto das ações desempenhadas pela MSU no Brasil e no mundo, é possível notar que a exportação e expansão do Management no País segue o padrão de uma ação organizada e orquestrada pelo governo norte-americano para exportar o desenvolvimento à EUA a outros países do mundo, principalmente durante os anos de 1950 e 1960. Não se trata, assim, de uma ação isolada. Para se ter uma ideia, o mesmo gestor na MSU coordenava as atividades dos projetos a respeito de Management no Brasil e das atividades envolvidas com a presença da universidade no Vietnam.

A MSU teve a função de apoiar o desenvolvimento e a expansão da capacidade de se ensinar Management no Brasil, oferecendo expertise técnica para a empreitada. O Artigo I do contrato IAAc-45 entre a MSU e o governo dos EUA estabelece com detalhes as funções da universidade em relação à EAESP e às demais instituições de ensino de Management no Brasil. No Artigo I consta:

1. Advise and assist the Foundation in instituting modern teaching methods at the school; 2. Advise and assist the Foundation in developing a curriculum for the school in developing programs for the courses to be taught at the school; 3. Provide guidance to Brazilian professors in their teaching courses at the school; 4. Undertake the teaching of courses at the School up to a maximum of 9 hours of class per week for each technician assigned by the college in Brazil under this agreement; 5 . Train Brazilian teaching assistants who may be assigned to work with the technicians assigned by the college in Brazil with the objective of preparing these assistants to take over the teaching of the course initially to be taught by the technicians assigned by the College; 6 . Advise and assist Getulio Vargas Foundation in the planning and implementation of a senior management training program for business and governament executives on a part-time non-degree basis; [...] B. The college [MSU] shall be responsible for furnishing competente technicians to perform the services provided for in Paragraph A above, and for paying all salaries, allowences, travel costs and other expenses necessary to the satisfactory performance of services provided for herein except as otherwise specifically provided in this Agreement [...] 3. The contractor shall be responsible for the technical competence of its personnel assigned to perform services in Brazil under this Agreement, and for satisfying itself with respect to their character, moral fitness, and suitability for assignment in Brazil in view of the condintions and cultural patterns that will encountered there; provided, that prior to the assignment of any individual to perform services under 
this Agreement in Brazil, the College will obtain the approval of the Institute shall submit biographic information on such individual requested by the Institute.

As funções da MSU estavam relacionadas com implementar e estabelecer uma escola de negócios no Brasil que deveria servir de centro difusor do Management no País. Para tanto, a MSU enviou uma missão de onze professores entre 1954 e 1958, que vieram ao País e ficaram por, aproximadamente, um período de 2 anos cada um deles - a maioria esteve lotada na EAESP-FGV. Houve, ainda, professores na UFRGS e na UFBA, e no curso de Engenharia da Produção na Escola Politécnica da Universidade de São Paulo. A MSU tinha o papel de recrutar, selecionar, enviar e gerir todas as questões associadas ao envio e à manutenção dos professores. Isso envolvia ações tais como: obter o certificado de que não tinham pendências com a Justiça dos EUA nem ligações com comunistas; visto na embaixada brasileira em Washington e, ainda, operacionalizar toda a mudança - móveis, utensílios domésticos e, até mesmo, carros eram enviados por navio para o Brasil. Tratava-se de uma operação bastante laboriosa, cheia de minúcias e detalhes. Os professores mantinham correspondências frequentes com a MSU e reportavam suas atividades no Brasil. Como vimos, a Fundação Ford (FF) financiou, junto com o CNPq, atividades relacionadas com a expansão do Management no Brasil. Na próxima sessão, trataremos do papel desempenhado pela FF na exportação e expansão do Management no Brasil.

\section{FUNDAÇÃO FORD}

A FF foi criada em 1936 por Henry Ford para evitar novos impostos estaduais sobre sua herança e, com isso, manter quase intacta a riqueza da família (ARNOVE e PINEDE, 2007). No início, a FF estava intimamente relacionada com os interesses da empresa na área de Detroit. Nesse período, as atividades da FF eram modestas em escala. No entanto, na década de 1940, a FF adquiriu $90 \%$ das ações da Ford Motor Company, e, assim, se tornou a fundação mais rica do mundo. 0 envolvimento de Henry Ford II foi decisivo ao nível de alto poder alcançado pela FF (SUTTON, 1987).

A FF é uma organização extremamente influente e desempenhou papel decisivo na reformulação da educação do Management nos EUA. Um de seus principais presidentes foi Thomaz (Tom) Carroll. Em 1959, Tom Carroll, então Vice-Presidente da FF, publicou um artigo no Journal of the Academy of Management (atual AMJ) intitulado "A Fundação expressa seu interesse na Educação em Management". Nesse trabalho, Carroll (1959) afirma que o interesse da FF na educação em Management vem de recomendações feitas por um comitê que estabeleceu, em 1948, as direções que a instituição deveria tomar e os recursos a serem alocados. Tal comitê sugeriu que a FF deveria direcionar seus recursos monetários "[...] para trabalhar para o bem-estar humano" (CARROLL, 1959, p. 155). Khurana e Spender (2012) argumentam que a FF e o governo dos EUA haviam notado a necessidade de se estabelecer uma base científica na formação em Management como um importante mecanismo por meio do qual os EUA iriam cumprir suas "[...] obrigações morais e para o desenvolvimento econômico" do pós-guerra (KHURANA e SPENDER, 2012, p. 7). Segundo Carroll, tratava-se de estabelecer "[...] a base inevitável do progresso na solução do problema de management, bem como de outros problemas humanos, como mitigar a tensão que aparece continuamente para ameaçar a paz mundial" (CARROLL, 1952, p. 552).

A preocupação da FF com a educação em Management também teve uma dimensão internacional. Em 1959, Carroll notou que a FF já patrocinava iniciativas de educação, nesta área, na Europa e na Ásia (CARROLL, 1959). Ele apontou, especificamente, que:

Estudantes estrangeiros vêm muitas vezes a instituições nos Estados Unidos para fazer sua pós-graduação. De fato, o crescente prestígio das nossas escolas de negócios provavelmente irá elevar o número de estrangeiros. Além disso, professores americanos irão desempenhar por algum tempo papel significativo na formação de professores e pessoal de pesquisa no exterior. (CARROLL, 1959, p. 164).

Como vimos, a FF acreditava estar incumbida de um "dever moral" de fomentar o desenvolvimento econômico (KHURANA e SPENDER, 2012). Tal suposto dever foi a justificativa ética para a construção de uma agenda internacional mais ampla.

Em 1956, Robert Aaron Gordon e James E. Howell foram convidados pela sede da FF para desenvolver um relatório de análise a respeito dos objetivos e métodos de ensino nas escolas de Management visando atender as exigências dos negócios e da sociedade (CARROLL, 1959). Produziram um relatório de 470 páginas denominado "Ensino em Management" 
(GORDON e HOWELL, 1959). O relatório continha a recomendação de uma "reforma ampla e drástica" na educação em Management nos EUA para torná-la mais "científica". De acordo com Khurana e Spender (2012), o objetivo da FF quanto à educação em Management era o de torná-la mais substancial academicamente e com uma orientação voltada para as necessidades da "gestão americana". Usando todo o seu poder financeiro nos EUA e no exterior, a FF difundiu um modelo de educação de negócios que exigia mais rigor disciplinar, maior ênfase na pesquisa quantitativa entre os docentes, uma seleção mais rigorosa dos alunos e treinamento sistemático para professores e alunos nas disciplinas de psicologia, sociologia, economia e estatísticas (KHURANA e SPENDER, 2012).

De acordo com Khurana, Kimura e Foucard (2011), a FF reformulou a educação em negócios nos EUA, tornou-a mais acadêmica e disciplinada, conduzindo-a em direção a uma base mais quantitativa e mais fundamentada em uma visão da economia clássica. Tom Carroll foi um dos principais patrocinadores dessa ideia (POOLEY e SOLOVEY, 2010). Acreditava-se que, ao aplicar a disciplina, o rigor e as leis da ciência para as organizações, seria possível construir uma ciência da gestão da mesma maneira como a engenharia projeta e constrói pontes (KHURANA e SPENDER, 2012).

O sucesso da FF na mudança do campo do ensino de Management levou Khurana, Kimura e Foucard (2011) a denominá-la uma "instituição dominante". Para os autores, tais organizações têm as seguintes características: são capazes de fazer intermediação entre diferentes setores institucionais e, além disso, podem legitimar ou estigmatizar organizações e/ou suas práticas e criam dependência de recursos com as principais organizações que estão tentando mudar. A FF atuou no pós-II Guerra no chamado "Terceiro Mundo", promovendo modelos e valores dos Estados Unidos por meio de uma versão específica de trabalho intelectual. A FF promoveu globalmente a criação de valores "científicos" dos EUA na academia de diferentes países.

A FF desempenhou papel crucial na solidificação da FGV EAESP em um momento no qual esta enfrentava dificuldades finan ceiras. No início, o suporte da FF parecia ser de fácil alcance. Em dezembro de 1956, o Conselho de Curadores da EAESP (MINUTAS DO CONSELHO DE CURADORES, setembro, 1956) teve conhecimento de que dois contatos pessoais haviam sido realizados com a FF para explorar as possibilidades de esta financiar a EAESP. Porém, em ambas as ocasiões, a resposta foi similar. Em conversa com um representante da FF em uma reunião na MSU, o representante da FF percebeu "[...] pequena chance de auxílio porque eles estavam concentrando no Oriente Médio, Sudeste da Ásia e África, por serem áreas mais próximas a países comunistas dominados" (MINUTAS DO CONSELHO DE CURADORES, setembro, 1956, p. 07).

Resposta similar foi dada ao diretor da MSU, por Thomas Carroll, à época vice-presidente da FF. As minutas do Conselho de Curadores (setembro, 1956) deixam claro que Carroll considerou não elegível a EAESP aos recursos da FF uma vez que "O Brasil não estava na zona de perigo" (Minutas do Conselho de Curadores, setembro, 1956, p. 07).

Na reunião de 1958 do Conselho de Curadores da EAESP (Minutas do Conselho de Curadores, setembro, 1958), ficou registrado que um membro do conselho escreveria para o ministro ("Butrick") no Departamento de Estado americano solicitando que este "levasse o problema até a Fundação Ford" (Minutas do Conselho de Curadores, janeiro, 1958, p. 22).

Coincidentemente ou não, em 1960, a Fundação Ford demonstra "grande interesse" (Minutas do Conselho de Curadores, março, 1960) em considerar um pedido de doação pela EAESP. Em março de 1961, o diretor de pesquisa da MSU visita a EAESP por dois meses (Minutas do Conselho de Curadores, março, 1961). O objetivo da visita era delinear uma proposta para fomentar a pesquisa acadêmica na EAESP. Esse fato foi percebido na EAESP como um passo crucial em direção ao estabelecimento de uma parceria com a FF. De acordo com os arquivos da EAESP (Minutas do Conselho de Curadores, maio, 1961), o vice-presidente da FF, senhor F. F. (sic) Hill, também visitou a escola em maio de 1961 e deixou claro para o diretor que a FF patrocinaria o desenvolvimento de atividades de pesquisa na EAESP, tanto que esta enviou um relatório e um plano para serem analisados pela FF. O representante da FF mencionou, até mesmo, o interesse da FF em disponibilizar para a EAESP cerca de $\$ 100,000$ na época (Minutas do Conselho de Curadores, maio, 1961). Na reunião do Conselho de Curadores, ao longo da discussão sobre tais números, um dos representantes brasileiros questiona o diretor se "a Fundação Ford estava realmente disposta em fazer uma contribuição tão alta para a escola [...]" (Minutas do Conselho de Curadores, maio, 1961, p. 5).

A quantia de dinheiro que a FF estava disposta a oferecer era substancial. Porém, isso somente ocorreria se a comunidade de negócios brasileira contribuísse também (Minutas do Conselho de Curadores, maio, 1961). Quando Ford foi informado 
de que o diretor estava presente, afirmou que ficaria "muito satisfeito em patrocinar a escola" (Minutas do Conselho de Curadores, maio, 1961, p. 16).

A FF financiou a tradução e a produção de livros-texto no campo de Management no Brasil (veja ALCADIPANI e COOKE, 2012 para uma análise) e também forneceu recursos para o desenvolvimento da capacidade de produção de pesquisas de qualidade internacional, ou seja, similar àquelas realizadas na instituição, nos EUA, mas houve problemas para atingir o objetivo (COOKE e ALCADIPANI, 2015).

Conforme apontamos anteriormente, a FF, em conjunto com o governo brasileiro, financiou projetos ligados à expansão do ensino do Management no Brasil. Os dois projetos mencionados faziam parte desse processo. Além disso, um projeto sob a responsabilidade de Donald Taylor, membro da missão de professores da MSU no Brasil, foi elaborado com o seguinte objetivo:

To determine the extent to which the discipline of business admininistration is institutionalized throughout the Brazilian higher education system; to identify elements in the institutionalizing process that may be useful in planning more effective use of resources in the diffusion of ideas; to expand education in business administration to the University of Rio Grande do Sul and the University of Bahia (HORN, 1985, p. 46).

Em consulta aos arquivos da FF em Nova York, encontramos referências a esforços por parte da instituição em termos financeiros e na parte de pessoal da MSU e da EAESP com o objetivo de auxiliar a UFRGS e a UFBA em relação ao ensino de Management. Memorando interno de 24 de junho de 1964 (A116) indica a necessidade da FF de bolsas de estudos para que professores da UFRGS e da UFBA, instituições que possuíam técnicos da MSU, realizassem estudos de pós-graduação na EAESP-FGV e voltassem para suas instituições de ensino. A ideia era forçar a FGV-EAESP a levar em consideração a sua posição como uma instituição de treinamento de professores. Em outro memorando interno de maio de 1972 (209:9) da FF, tem-se o registro de que a EAESP já havia treinado 10 professores para a região Nordeste, e que a Universidade Federal de Pernambuco havia considerado se tornar um centro de treinamento na área de Management para a região, e com a presença de professores da EAESP.

Um memorando interno de outubro de 1973 (213:18) indica que havia poucas possibilidades de a EAESP auxiliar na expansão do Management para a região Nordeste e outras áreas do País. Indicava, ainda, que as tentativas da EAESP de criar parcerias com outras instituições para a qualificação de seus quadros em Management, de fato, não obtiveram sucesso. Os técnicos da FF indicaram que isso não ocorreu porque a região Nordeste era "atrasada, ainda uma área presa em seu passado colonial" e que as empresas, quando precisavam de pessoal na região, traziam trabalhadores do Sul como se esses fossem "expatriados". O memorando considerou, ainda, que em 1963, dentro de um acordo com a USAID e a MSU, a EAESP firmou parcerias com a UFRGS e com a UFBA, mas que essas universidades nunca renderam os frutos desejados e que os acadêmicos treinados em São Paulo foram mal preparados.

A FF teve o papel de financiar algumas atividades ligadas à difusão do Management no Brasil. Especificamente essa fundação buscou catalisar a expansão do ensino de gestão para outras áreas geográficas e também conduzir a produção acadêmica no Brasil seguindo o tipo de pesquisa feito nos EUA. A FF entrou fundamentalmente com recursos financeiros concedidos sob a condição de que os objetivos que a Fundação Ford desejava para o ensino do Management fossem seguidos no Brasil. O recurso financeiro era uma maneira de criar a possibilidade de se exercer influência nos rumos estratégicos do ensino do Management no Brasil. Periodicamente, consultores da FF vinham ao Brasil auditar se os recursos financeiros estavam sendo empregados nos fins estabelecidos.

\section{DISCUSSÕES E CONCLUSÕES}

Vimos neste texto que a educação em Management é exportada para o Brasil como uma ação deliberada e coordenada de algumas instituições dos EUA (governo, universidades e fundações), em parceria com o governo brasileiro e uma fundação do País, a FGV. Escolhemos por centrar nossa análise no papel desempenhado por instituições norte-americanas, pois elas 
desempenharam papel crucial em fornecer auxílio técnico e financeiro para a empreitada. Em estudo anterior, discutimos o papel da Guerra Fria nesse processo (ALCADIPANI e BERTERO, 2012). Vale frisar que esse auxílio foi, inclusive, solicitado pelo governo do Brasil.

Neste texto, permenorizamos a análise e trouxemos como pano de fundo as relações entre Brasil e EUA. Vimos que no período em que o ensino do Management é exportado e se expande no Brasil, o País passava por um período político bastante conturbado. Entre 1953 e 1966, tivemos o suicídio de Getúlio Vargas, a renúncia de Jânio Quadros e a deposição de João Goulart. Excetuando-se o período do milagre econômico no governo Juscelino, a economia brasileira esteve com problemas sérios e a relação com os EUA, conturbada. Conforme destacamos, havia pressões expressivas para que os EUA fornecessem mais ajuda e cooperação ao Brasil. Percebemos, ainda, uma grande desconfiança dos EUA com relação a presidentes brasileiros, mas que não impediu a cooperação para a exportação e a expansão do ensino do Management no Brasil. Vimos, assim, que a primeira instituição com o papel de viabilizar a exportação e expansão do Management no Brasil foi a representação diplomática dos EUA no País. Ela cumpriu um papel de municiar o governo dos EUA com informações sobre o Brasil e também de proteger os interesses dos EUA no País, servindo, ainda, de suporte às operações da missão da MSU nos seus diferentes projetos.

Apesar das turbulências nas relações entre Brasil e EUA, provavelmente a cooperação em ensino e pesquisa ocorreu devido ao fato de haver um grande interesse dos EUA em parcerias acadêmicas durante esse período, principalmente devido à Guerra Fria, conforme argumentamos em Alcadipani e Bertero (2012). A MSU esteve envolvida em 150 projetos de cooperação técnica internacional em diferentes países do mundo, em inúmeras áreas do conhecimento - isso sem falar nas demais universidades do país. Havia, nos EUA, àquela época, uma verdadeira indústria de cooperação internacional acadêmica com muitos recursos disponíveis para esse tipo de projeto. Vimos anteriormente os valores expressivos gastos pelos EUA no projeto de exportação e expansão do ensino do Management em nosso país. Um ponto que chama atenção é o fato de que os EUA financiavam universidades norte-americanas com a contratação de técnicos e professores dos EUA para realizar as tarefas no Brasil. Com isso, os recursos financeiros ficaram em posse dos EUA o tempo todo e acabaram por financiar as universidades Norte Americanas. Dentro do montante investido, pouco veio para o Brasil. Notamos, ainda, que o projeto sobre o Management não era o único realizado pela MSU no Brasil. Isso indica que suas atividades no País foram maiores em escopo e profundidade.

A MSU forneceu professores que formaram uma missão que veio ao Brasil com o intuito de conduzir atividades para desenvolver o ensino do Management no País. Esses professores estiveram presentes na FGV EAESP, na UFRGS e na UFBA. O ensino do Management já era desempenhado na ESAN, mas essa instituição era uma criação de um entusiasta do ensino do Management e ficou bastante circunscrita ao espaço da Escola. Foi apenas quando contou com o apoio do governo e de fundações norte-americanas que o Management ganhou destaque no Brasil e se expandiu para o Sul e Nordeste. Assim, a MSU foi a instituição norte-americana que terminou por ter a responsabilidade de exportar e expandir o Management no País. Vimos acima que isso foi o resultado de um processo longo e que exigiu muitos recursos. A Ford Foundation entrou quando o ensino do Management já tinha destaque no País e forneceu auxílio financeiro para poder contribuir ainda mais na consolidação da empreitada. Ela fornecia apoio financeiro, que foi bem mais limitado do que o do governo dos EUA, em troca de tentar influenciar os rumos que o ensino do Management deveria seguir. Vimos, todavia, que a FF não obteve muito sucesso em suas ações no Brasil. De modo geral, a representação dos EUA no Brasil, a MSU e a FF formaram uma rede que permitiu a exportação e a expansão do Management para o nosso país.

Neste texto, aprofundamos a questão da exportação e expansão do ensino do Management para o Brasil ao contextualizarmos essa ação dentro das relações Brasil-EUA da época. Além disso, mostramos detalhadamente o papel das instituições norte-americanas nesse processo e trazemos ainda a questão da expansão desse ensino para o Sul e o Nordeste do Brasil como resultado de apoio e pressões dos EUA para que isso acontecesse. Em próximos artigos, buscaremos pormenorizar as ações que permitiram a expansão do ensino do Management para a UFRGS e a UFBA. 


\section{REFERÊNCIAS}

ALCADIPANI, R.; BERTERO, C. O. A Guerra Fria e o ensino do Management no Brasil: o Ponto IV e a Escola de Administração de Empresas de São Paulo da Fundação Getulio Vargas (FGV-EAESP). In: ENCONTRO DE ENSINO E PESQUISA EM ADMINISTRAÇÃO E CONTABILIDADE, 3., 2011, João Pessoa. Anais... João Pessoa: ANPAD, 2011.

ALCADIPANI, R.; BERTERO, C. O. Guerra Fria e o ensino do Management no Brasil: o caso da FGV-EAESP. Revista de Administração de Empresas, v. 52, n. 3, p. 284-299, 2012.

ALCADIPANI, R.; CALDAS, M. Americanizing brazilian management. Critical Perspectives on International Business, v. 8, n. 1, p. 37-55, 2012.

ALCADIPANI, R.; COOKE, B. The case of the missing textbooks, or textbook case? A brazilian micro-history of the transfer of Management knowledge, 2012. mimeo.

ALCADIPANI, R.; ROSA, A. R. From global management to local management: Latin American perspectives as a counter-dominant management epistemology. Canadian Journal of the Administrative Sciences, v. 28, p. 453-466, 2011.

ANDERSON, D. Management education in developing countries: the brazilian experience. London: Westview Press, 1987.

ARNOVE, R.; PINEDE, N. Revisiting the 'Big Three' Foundations. Critical Sociology, v. 33, n.3, p. 389-442, 2007.

BARROS, A. N. Uma narrativa sobre a história dos cursos de Administração da Face-UFMG: às margens do mundo e à sombra da FGV? 186f. 2013. Tese (Doutorado em Administração). Programa de Pós-Graduação, FACE-UFMG, 2013.

BARROS, A. N.; CARRIERI, A. P. Ensino superior em Administração entre os anos 1940 e 1950: uma discussão a partir dos acordos de cooperação Brasil-Estados Unidos. Cad. EBAPE.BR, v. 11, n. 3, p. 256-273, 2013.

BERTERO, C. Ensino e pesquisa em Administração. São Paulo: Thompson Learning, 2006.

BOOTH, C.; ROWLINSON, M. Management and Organizational History: prospects. Management \& Organizational History, v. 1, p. 5-30, 2006.

CALDAS, M. Santo de casa não faz milagre. In: MOTTA, F. P.; CALDAS, M. (Coord.). Cultura Organizacional e Cultura Brasileira. São Paulo: Atlas, 1997.

CALDAS, M.; ALCADIPANI, R. Americanização e pós-colonialismo: a gênese da referência norte-americana na cultura e gestão no Brasil. In: CHANLAT, J. F. et al. (Org.). Análise das organizações - perspectivas latinas. Porto Alegre: Editora da UFRGS, 2006.

CARROLL, T. The need for increasing convergence of Management theory and practice. Southern Economic Journal, v. 18, n. 4, p. 552 558, 1952.

CARROLL, T. A foundation expresses its interest in higher education for business management. The Journal of the Academy of Management, v. 2, n.3, p. 155-165, 1959.

CLARK, P.; ROWLINSON, M. The treatment of history in organisation studies: towards an 'historic turn'? Business History, v. 46, n.3, p. 331-352, 2004.
COOKE, B. Writing the left out of management theory: the historiography of the management of change. Organization, v. 6, n. 1, p. 81-106, 1999.

COOKE, B. The denial of slavery in Management studies. Journal of Management Studies, v. 40, n. 8, p. 1835-1918, 2003.

COOKE, B.; ALCADIPANI, R. Towards a global history of management education: the case of the foundation and the São Paulo School of Business Administration. Brazil Acad Manag Learn Education, 2015. No prelo.

COSTA, A.; BARROS, D.; CARVALHO, J. A dimensão histórica dos discursos acerca do empreendedor e do empreendedorismo. Rev. Adm. Contemporânea [online], v. 15, n. 2, p. 179-197, 2011. Disponível em: <http://www.scielo.br/pdf/rac/v15n2/v15n2a02.pdf>. Acesso em: 4 jan. 2012.

COSTA, A.; BARROS, D.; MARTINS, P. Perspectiva histórica em administração: novos objetos, novos problemas, novas abordagens. Revista de Administração de Empresas, v. 50, n. 3, p. 288-299, 2010.

COVRE, M. L. M. A formação e a ideologia do administrador de empresa. São Paulo: Editora Vozes, 1981.

CURADO, I. B. Pesquisa historiográfica em Administração: uma proposta metodológica. In: ENCONTRO NACIONAL DA ASSOCIAÇÃO NACIONAL DE PÓS-GRADUAÇÃO E PESQUISA EM ADMINISTRAÇÃO, 25., 2005, Foz do Iguaçu. Anais... Foz do Iguaçu: ANPAD, 2005.

D'ARAUJO, M. Fundação Getulio Vargas: concretização de um ideal. Rio de Janeiro: Editora da FGV, 1999.

DUREPOS, G. A. J.; MILLS, T.; MILLS, J. H. Tales in the manufacture of knowledge: writing a company history of Pan American World Airways. Management \& Organizational History, v. 3, n. 1, p. 63-80, 2008.

DUREPOS, G.; MILLS, A. J.; WEATHERBEE, T. G. Theorizing the past: realism, relativism, relationalism and the reassembly of Weber. Management \& Organizational History, v. 7, n. 3, p. 267-281, 2012.

FISCHER, T. O Ensino de Administração Pública no Brasil - os ideais de desenvolvimento e as dimensões da racionalidade. São Paulo: FEA-USP, 1984.

GANTMAN, E.; RODRIGUES, C. Notas sobre la evolución del conocimiento administrativo em la República Argentina y su comparación con el caso español (1913-2007). Cad. EBAPE.br, v. 6, n. 4, p. 2-22, 2008.

GERSTLE, G. Working-class americanism: the politics of labour in a textile city, 1914-1960. Cambridge: Cambridge University Press, 1989.

GORDON, R. A.; HOWELL, J. E. Higher Education for Business. New York: Columbia University Press, 1959.

IGGERS, G. Historiography in the twentieth century: from scientific objectivity to the postmodern challenge. Hanover: University Press of New England, 1997.

JACQUES, R. History, historiography and organization studies: the challenge and the potential. Management \& Organizational History, v. 1, n. 1, p. 31-49, 2006.

KHURANA R. From higher aims to hired hands: the social transformation of american business schools and the unfulfilled promise of Management as a profession. Princeton: Princeton University Press, 2007. 
KHURANA, R.; KIMURA, K.; FOURCAD, M. How foundations think: the Ford Foundation as a dominating institution in the field of american business schools. [Working Paper Number: 11-070]. Boston: Harvard Business School, 2011.

KHURANA, R.; SPENDER, J. C. Herbert A. Simon on what ails business schools: more than a problem in organizational design. Journal of Management Studies, v. 49, n. 3, p. 1-21, 2012.

MARCH, J. Parochialism in the evolution of a research community: the case of organization studies. Management and Organization Review, v. 1, n. 1, p. 5-22, 2007.

MILLS, A.; MILLS, J. H. Digging Archeology: Postpositivist Theory and Archival Research in Case Study Development. In: PIEKKARI, R,; Welch, C. (Eds.). Rethinking the case study in international business research. Northampton, MA: Edward Elgar Publishing, 2011, p. 342-360.

MILLS, A.; YUE, A. Historiography and the past in business studies. In BRYMAN, A. et al. Business Research Methods (Canadian Edition). Don Mills, Ontario: Oxford, 2011.

POOLEY, J.; SOLOVEY, M. Marginal to the Revolution: the Curious Relationship between Economics and the Behavioral Sciences Movement in Mid-Twentieth-Century America. History of Political Economy, v. 42, n.1, p. 199-233, 2010.

SEGNINI, L. R. P. Ferrovia e ferroviários: uma contribuição para a análise do poder disciplinar na empresa. São Paulo: Autores Associados/Cortez, 1982.
SERVA, M. A Importação de metodologias administrativas no Brasil - uma análise semiológica. Rev. Adm. Pública, v. 26, n. 4, p. 128144, 1992.

SUTTON, F. The Ford Foundation: the early years. Daedalus, v. 116, n. 1, p. 41-91, 1987.

TOTA, A. O imperialismo sedutor - a americanização do Brasil na época da Segunda Guerra. São Paulo: Cia das Letras, 2000.

ÜSDIKEN, B.; ÇETIN, D. From betriebswirtschaftslehre to human relations: turkish management literature before and after the Second World War. Business History, v. 43, n. 2, p. 99-124, 2001.

VALE, M. P. E. M. Institucionalização do ensino em administração de empresas na cidade de São Paulo: um estudo de caso sobre a Faculdade de Economia, Administração e Contabilidade da Universidade de São Paulo (FEA-USP). 144f. 2012. Dissertação (Mestrado em Administração). Programa de Pós-Graduação em Administração de Empresas da FGV-EAESP, São Paulo, FGV, 2012.

VIZEU, F. Management no Brasil em perspectiva histórica: o projeto do IDORT nas décadas de 1930 e 1940. São Paulo: FGV-EAESP, 2008.

VIZEU, F. Potencialidades da análise histórica nos estudos organizacionais brasileiros. Revista de Administração de Empresas, v. 50, n. 1 , p. $36-46,2010$

WARREN, J. The past and its presenters: an introduction to issues in historiography. Oxford: Hodder \& Stoughton, 1998.

Rafael Alcadipani

PhD em Business Administration pela Manachester Business School (Reino Unido); Professor Adjunto do Departamento de Administração Geral e Recursos Humanos da Escola de Administração de Empresas de São Paulo da FGV. Brasil. E-mail: rafael.alcadipani@fgv.br

Carlos Osmar Bertero

PhD em Management pela Cornell University (Estados Unidos); Professor Titular Departamento de Administração Geral e Recursos Humanos da Escola de Administração de Empresas de São Paulo da FGV. Brasil. E-mail: carlos.bertero@fgv.br 